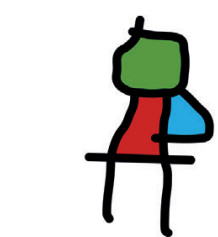

SEICAIP

\section{Allergologia et immunopathologia}

Sociedad Española de Inmunología Clínica, Alergología y Asma Pediátrica

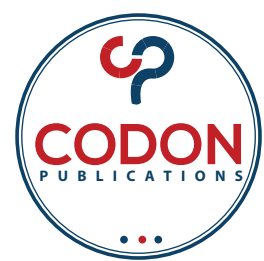

ORIGINAL ARTICLE

OPEN ACCESS CC)(1) (2)

\title{
Association between the type of allergen and T-helper 2 mediated inflammation in allergic reactions: a systematic review and a meta-analysis
}

\author{
Elina P. Nepolo ${ }^{a}$, Bongani B. Nkambule ${ }^{b *}$, Phiwayinkosi V. Dludla ${ }^{c, d}$, Fransina. Ndevahoma ${ }^{a}$, \\ Tawanda M. Nyambuyaa,b*
}

${ }^{a}$ Department of Health Sciences, Faculty of Health and Applied Sciences, Namibia University of Science and Technology, Windhoek, Namibia

${ }^{b}$ School of Laboratory Medicine and Medical Sciences, College of Health Sciences, University of KwaZulu-Natal, Durban, South Africa

'Department of Life and Environmental Sciences, Polytechnic University of Marche, Ancona, Italy

${ }^{d}$ Biomedical Research and Innovation Platform, South African Medical Research Council, Tygerberg, South Africa

Received 22 June 2021; Accepted 19 October 2021

Available online 1 January 2022

\section{KEYWORDS \\ Allergen; \\ interleukin-4; \\ interleukin-5; \\ inflammation; \\ T-helper 2 cells; \\ type 1 \\ hypersensitivity}

\begin{abstract}
Objectives: To determine whether the levels of T-helper $\left(\mathrm{T}_{\mathrm{H}}\right) 2$ cytokines (interleukin (IL)-4 and IL-5) in allergic reactions are allergen dependent and evaluate the impact of various treatment strategies on the levels of these cytokines.

Methods: The PubMed search engine was used from inception until January 2021. The random-effects residual maximum likelihood model was performed, and effect sizes were estimated using the Hedge's $g$ statistic. All data analysis was performed using STATA 16.0 (StataCorp LP, TX, USA).

Results: Fourteen studies reporting on 794 participants were included in this study. House dust mite was associated with eliciting a stronger immune response mediated by both IL-4 and IL-5 when compared to pollen. Whereas a mixture of house dust mite and pollen was associated with IL-4-weighted inflammation. Comparisons of IL-4 and IL-5 levels amongst the allergens showed significant differences. The treatment with anti-corticosteroids or allergen-specific immunotherapy was effective in normalising the $\mathrm{T}_{\mathrm{H}} 2$ responses and alleviating allergy symptoms.

Conclusion: $T_{H} 2$-mediated inflammation in allergic reactions is allergen-dependent. Therefore, the type of allergen should be considered when using cytokine-targeting biologics in allergic reactions.

(c) 2022 Codon Publications. Published by Codon Publications.
\end{abstract}

*Corresponding authors: Tawanda M. Nyambuya and Bongani B. Nkambule, Private Bag 13388 Windhoek, Namibia and P/Bag X3, Congella, Durban, 4013, South Africa. Email addresses: mnyambuya@nust.na and nkambuleb@ukzn.ac.za 


\section{Introduction}

A balance between T-helper $\left(T_{H}\right) 1$ and $T_{H} 2$ immune responses is important in modulating inflammation and maintaining immune homeostasis. ${ }^{1}$ An alteration in the $T_{H} 1 /$ $\mathrm{T}_{\mathrm{H}} 2$ ratio is a hallmark of several immune-mediated diseases, including immediate hypersensitivity disorders. ${ }^{2,3}$ In fact, allergic reactions are characterised by an exacerbated $\mathrm{T}_{\mathrm{H}}$ 2-skewed immune response and the manifestation of allergic symptoms such as atopic dermatitis, allergic rhinitis and asthma. ${ }^{4,5}$ Notably, the resulting inflammatory milieu is modulated by interleukin (IL)- 4 and IL-5, the two major cytokines that mediate $\mathrm{T}_{\mathrm{H}} 2$-mediated inflammation. ${ }^{6}$ The aggravated elevation of these cytokines is associated with increased activation of B-cells, eosinophils, basophils and the secretion of immunoglobulin (Ig)G and IgE in allergic reactions. ${ }^{7-9}$ As a result, several current therapeutic strategies aim to alleviate these symptoms and ameliorate $\mathrm{T}_{\mathrm{H}}$ 2-mediated inflammation. ${ }^{10-13}$

Although allergic responses are characterized by a predominant $\mathrm{T}_{\mathrm{H}} 2$ response, there are some discrepancies with regards to the levels of IL- 4 and IL- 5 in patients with allergies. For instance, the allergic responses in patients hypersensitive to house dust mite (HDM) are associated with elevated serum IL-4 and IL-13- levels. ${ }^{14,15}$ Whereas increased IL-5 levels have been reported in patients allergic to pollen. ${ }^{16} \mathrm{~A}$ comparison between patients allergic to HDM and pollen showed that the levels of IL-5 are in fact elevated in the former group. ${ }^{17}$ In patients allergic to peanuts, the immune response is skewed towards IL-4, ${ }^{10}$ albeit others suggested it to be IL-5-weighted. ${ }^{18}$ Therefore, these inconsistencies suggest that the immune responses in allergic reactions may be dependent on the type of allergen.

The use of allergen-specific immune therapy, antihistamines, and corticosteroids in allergic reactions has been to a greater extent successful in alleviating $\mathrm{T}_{\mathrm{H}} 2$-mediated inflammation and the associated symptoms, albeit poor efficacy due to various factors such as age and obesity has been noted in some patients. ${ }^{19,20}$ Consequently, cytokine-targeting biologics are currently being explored as an alternative treatment approach in allergic reactions. ${ }^{11,21,22}$ In this systematic review and meta-analysis, we aimed at assessing available literature reporting on $\mathrm{T}_{\mathrm{H}} 2$-mediated inflammation in allergic reactions. The primary objective of this study was to investigate whether the levels of IL-4 and IL-5, the key cytokines that mediate inflammation in an allergic response, are allergen-dependant. The secondary objective was to assess the impact of treatment on the levels of these cytokines.

\section{Methods}

This systematic review and meta-analysis was prepared following the preferred reporting items for systematic reviews and meta-analysis (PRISMA) guidelines. ${ }^{23} \mathrm{~A}$ protocol was designed and agreed upon by all authors before conducting the qualitative and quantitative synthesis (Appendix 1). The protocol was however not registered. We conducted a comprehensive and systematic search of available literature to answer the following research question: are $\mathrm{T}_{\mathrm{H}} 2$ immune responses in allergic reactions influenced by the type of allergens?

\section{Sources of evidence and search strategy}

A comprehensive search was designed and independently conducted by two reviewers (EPN and TMN) using the PubMed search engine from inception until January 2021. The following search terms were used to retrieve all relevant studies; “allergy", "allergens", “IL-4, “IL-5", “T 2 " and "type 1 hypersensitivity". Moreover, grey literature and the reference lists of included studies were scanned to identify any additional studies. No language restrictions were applied on the search strategies, and a third reviewer (BBN) was consulted for arbitration in cases of disagreements. A detailed MEDLINE search strategy on PubMed search engine is shown in Supplementary Table S1.

\section{Eligibility criteria and study selection}

Two independent investigators (EPN and FN), with the help of third reviewer (TMN), screened all titles and abstracts of the retrieved studies. Studies were included if they reported on the levels of both IL- 4 and IL-5 in patients of all ages with allergies.

\section{Inclusion and exclusion criteria}

The following PICO was used in the inclusion criteria:

Participants: patients of all ages with allergies

Intervention: none

Comparisons: healthy individuals without allergies (controls).

Outcome: $\mathrm{T}_{\mathrm{H}}$ 2-mediated inflammation

We excluded reviews, case studies, letters to the editor and animal studies from this systematic review and meta-analysis. In addition, studies that were not relevant to the topic of interest or with no suitable comparator were also excluded.

\section{Data extraction and management}

Two independent reviewers (EPN and FN) extracted detailed study information and characteristics using a predefined data extraction form adapted from the Cochrane Consumers and Communication Review Group data extraction for included studies template. ${ }^{24}$ The extracted data consisted of names of authors, publication year, study design, study size, age and gender, types of allergens, IL-4 and IL-5 levels and the main findings of each study. The extracted data items were verified by $\mathrm{FN}$, and disagreements were resolved through discussions or by consulting the third reviewer (TMN).

\section{Assessment of risk of bias}

The risk of bias in all included studies was independently assessed by two reviewers (EPN and FN) using the modified Downs and Black checklist. ${ }^{25}$ The checklist consists 
of four domains, reporting bias, external validity, internal validity and selection of bias. The studies were rated as follows: excellent (a score between 24-27), good (score of 19-23), fair (score of 13-18) or poor (score of <12). A third reviewer (PVD) was consulted for arbitration in cases of disagreements. The Cohen's kappa scores were used to measure inter-rater reliability, and a score of 0.00 was considered poor, slight (0.01-0.20), fair (0.21-0.40), moderate (0.41-0.60), substantial (0.61-0.80) and perfect $(0.81-1.00){ }^{26}$

\section{Statistical analysis}

The mean and standard deviation for each effect measure was either extracted or estimated using GetData Graph Digitizer software or calculated from the median range using Hozo et al.'s method. ${ }^{27}$ The continuous outcome was reported as standardised mean differences and 95\% confidence interval $(\mathrm{Cl})$, and effect sizes were estimated using the Hedge's $g$ statistic to correct for small study bias. A random-effects residual maximum likelihood (REML) model was performed to minimise the bias of variance estimator. A sensitivity analysis was performed based on study designs to evaluate the robustness of the reported overall effect estimates. A P value of $<0.05$ was considered significant. All data analysis was performed using STATA 16.0 (StataCorp LP, TX, USA).

\section{Results}

\section{Selected studies}

The search identified a total of 71 studies which were assessed for eligibility. A total of 50 studies were excluded at the abstract screening stage as these were not describing findings related to the outcomes of the present study. The remaining 21 studies were assessed for eligibility using full texts and a total of seven citations were excluded because five were not relevant to the topic of interest, while two studies had no suitable comparators. As a result, only 14 studies met the inclusion criteria and were included in this systematic review and meta-analysis (overall agreement, 97.62\%; kappa=0.95), and only eight were included in the quantitative synthesis (Figure 1).

\section{Characteristics of included studies}

The included studies consisted of two randomised controlled trials (RCTs), three non-RCTs and nine observational studies published in peer-reviewed journals between 1997 and 2017. Of these studies, four were from Asia, 12,15,28,29 three from Europe, ${ }^{13,30,31}$ five from North America ${ }^{10,14,16,18,32}$ and two from Oceania. ${ }^{17,33} \mathrm{~A}$ total of 794 participants with a male/female ratio of 0.53 were included in this systematic review and meta-analysis. Of which, 565 participants had allergies, and 229 were healthy controls. The cohort consisted of $64 \%$ of the participants being children (Table 1) and $36 \%$ adults (Table 2 ) with an average age of $11.10 \pm 4.44$ years and $44.03 \pm 17.45$ years, respectively. A total of $26 \%$ of patients from the allergic group were allergic to pollen, $29 \%$ to HDM, $4 \%$ to peanut and $13 \%$ consisted of a mixture of patients allergic to pollen and HDM, whilst $28 \%$ were not specified.

\section{Study quality and risk bias}

The median score range of included studies was 15 (14-24) out of a possible score of 27 (Supplementary Table S2)..$^{14-24}$ All studies were rated as fair ${ }^{10,12,13-18,28,31-33}$ except for one study which was scored as good. ${ }^{29}$ Thus, the included studies had a moderate risk of bias. Assessments based on the four domains showed that the included studies had a low risk of reporting bias with a median score of 7.5 (6-10) out of the possible score of 11 (overall agreement, 92.85\%; kappa=0.86) and internal validity bias, a median of 4 (3-6) out of the possible score of 7 (overall agreement, 92.85\%; kappa $=0.86)$. However, the studies had high risk of external validity bias with a median of $1(0-2)$ out of the possible score of 3 (overall agreement, $88.10 \%$; kappa $=0.76)$ and selection bias with a median of $2.5(2-6)$ out of the possible score of 6 (overall agreement, 97.62\%; kappa $=0.95)$.

\section{The levels of IL-4 in patients with allergies are influenced by the type of allergen}

Most of the included studies (36\%) involved patients allergic to pollen, followed by those allergic to HDM (29\%) and peanuts (14\%). Interleukin-4 shares common receptor and functional properties with IL-13, ${ }^{34}$ and $57 \%$ of the included studies reported elevated levels of both cytokines. Allergic responses irrespective of the allergens were reported to be IL-4-weighted in $36 \%$ of the included studies, ${ }^{10,14,15,29,32}$ whereas a few reported undetectable low levels. ${ }^{17,18}$ Notably, the levels of IL- 4 and IL-13 were associated with those of allergen-specific $\operatorname{lgE}^{10,31}$ and disease severity. ${ }^{12}$ We performed a subgroup analysis based on the type of allergen and participants' characteristics. The test for subgroup differences was only significant in the allergen subgroup ( $P=0.01)$, which showed that the type of allergen in these patients influenced the levels of IL-4 and had an impact on the reported effect size. Studies that included patients allergic to HDM (Hedges' g: 0.68 [95\% confidence interval [CI]: $0.12,1.24], \mathrm{P}=0.018$ ) and those that involved a mixture of patients allergic to pollen and HDM (Hedges' g: 1.02 [95\% Cl: 0.39, 1.65], $\mathrm{P}=0.002$ ) had a large increase in IL-4 levels than healthy controls. However, pollen $(\mathrm{P}=$ $0.579)$ and unspecified allergens $(P=0.065)$ did not affect the reported effect measure of $\mathrm{T}_{\mathrm{H}} 2$ immune responses (Figure 2). We further compared the standardised means of IL-4 levels in each antigen. Notable differences were observed in pollen versus HDM $(P=0.0430)$ versus pollen and HDM ( $P=0.0009)$ and versus unspecified antigens $(P=$ $0.0002)$. In addition, HDM vs. unspecified antigens $(P=$ 0.0024) (Supplementary Table S3). We performed a sensitivity analysis based on the study design. Notably, Hedges's $g$ did not change direction nor the magnitude of effect size (Supplementary Table S4). 


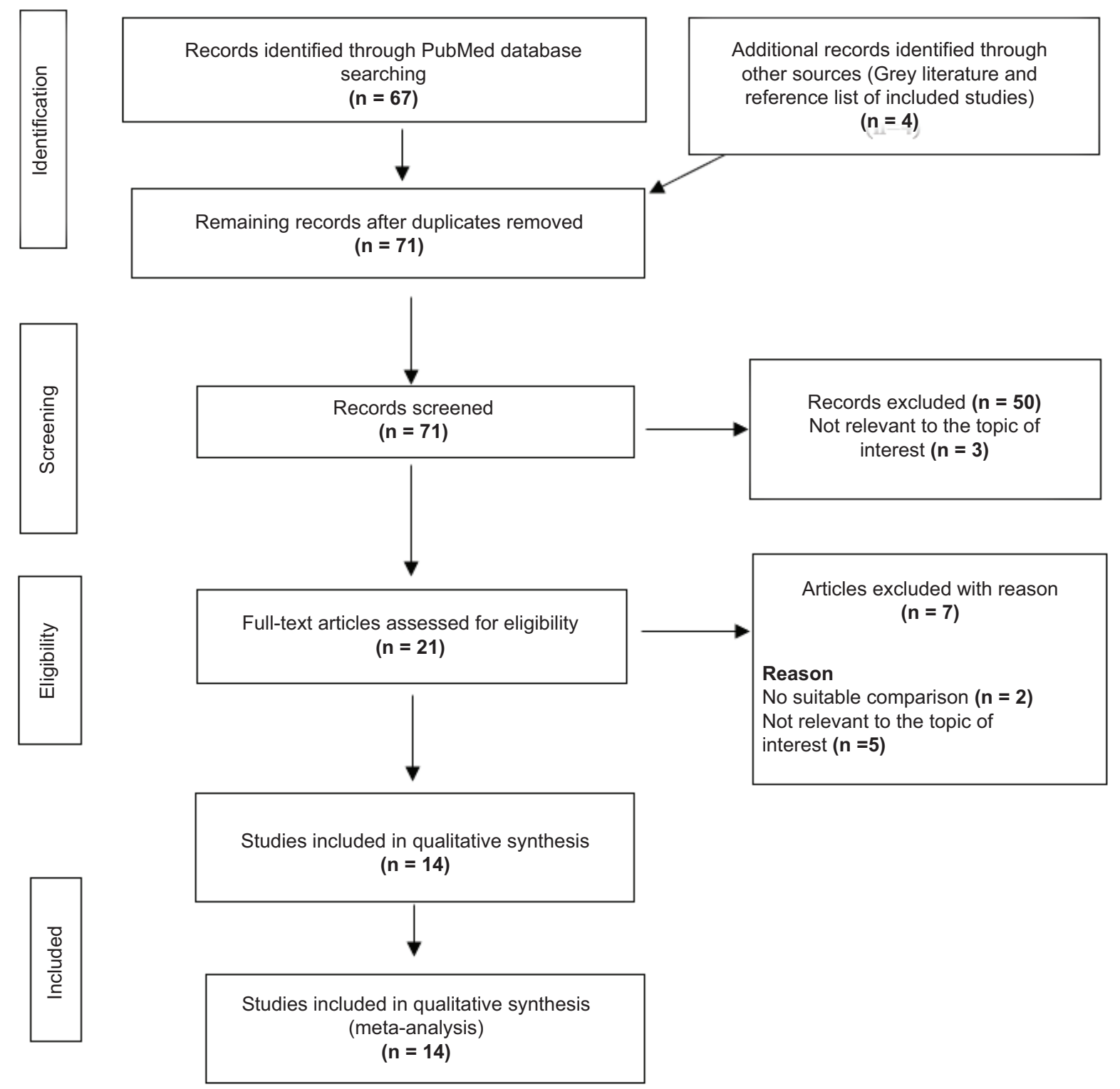

Figure 1 PRISMA flow diagram showing the study selection process.

\section{Different types of allergen influence IL-5 levels in patients with allergies}

A total of $43 \%$ of the included studies ${ }^{12,16-18,28,33}$ reported IL-5-weighted immune responses in patients with allergy, whereas $14 \%$ reported on comparable or undetectably low levels. ${ }^{14,15}$ The elevated levels of IL-5 in patients with allergy were also associated with increased levels of $\operatorname{lgE},{ }^{17}$ eosinophils ${ }^{12,13}$ and disease severity. ${ }^{28,33}$ A comparison between patients allergic to HDM and pollen showed a significant increase in IL-5 levels in the former group. ${ }^{17} \mathrm{We}$, therefore, performed a meta-analysis to assess the levels of IL-5 in patients with allergies. Similarly to IL-4, the quantitative synthesis of included studies revealed that IL-5 levels are dependent on the type of allergen and not participants' characteristics. The test for subgroup differences in the allergen subgroup was significant $(P=0.01)$, suggesting that allergens modified the reported effect size of $\mathrm{T}_{\mathrm{H}} 2$ immune responses. Studies involving participants allergic to HDM (Hedges' g: 0.80 [95\% Cl: 0.02, 1.59], $\mathrm{P}=0.044$ ) and unspecified allergens (Hedges' g: 2.04 [95\% Cl: 1.10, 2.98], $P<0.01$ ) had a large increase in the levels of IL-5 versus healthy controls (Figure 3 ). However, studies including patients allergic to pollen $(P=0.857)$ or a mixture of patients allergic to pollen and HDM $(P=0.082)$ did not modify the effect estimate. Comparisons of standardised means of IL-5 levels amongst the allergens showed significant differences in unspecified antigens versus pollen $(\mathrm{P}<$ $0.0001)$ versus HDM $(P=0.0086)$ versus pollen and HDM $(P=$ 0.0001) (Supplementary Table S3). The sensitivity analysis showed that only the cohort study changed the direction of the effect size (Supplementary Table S4). 


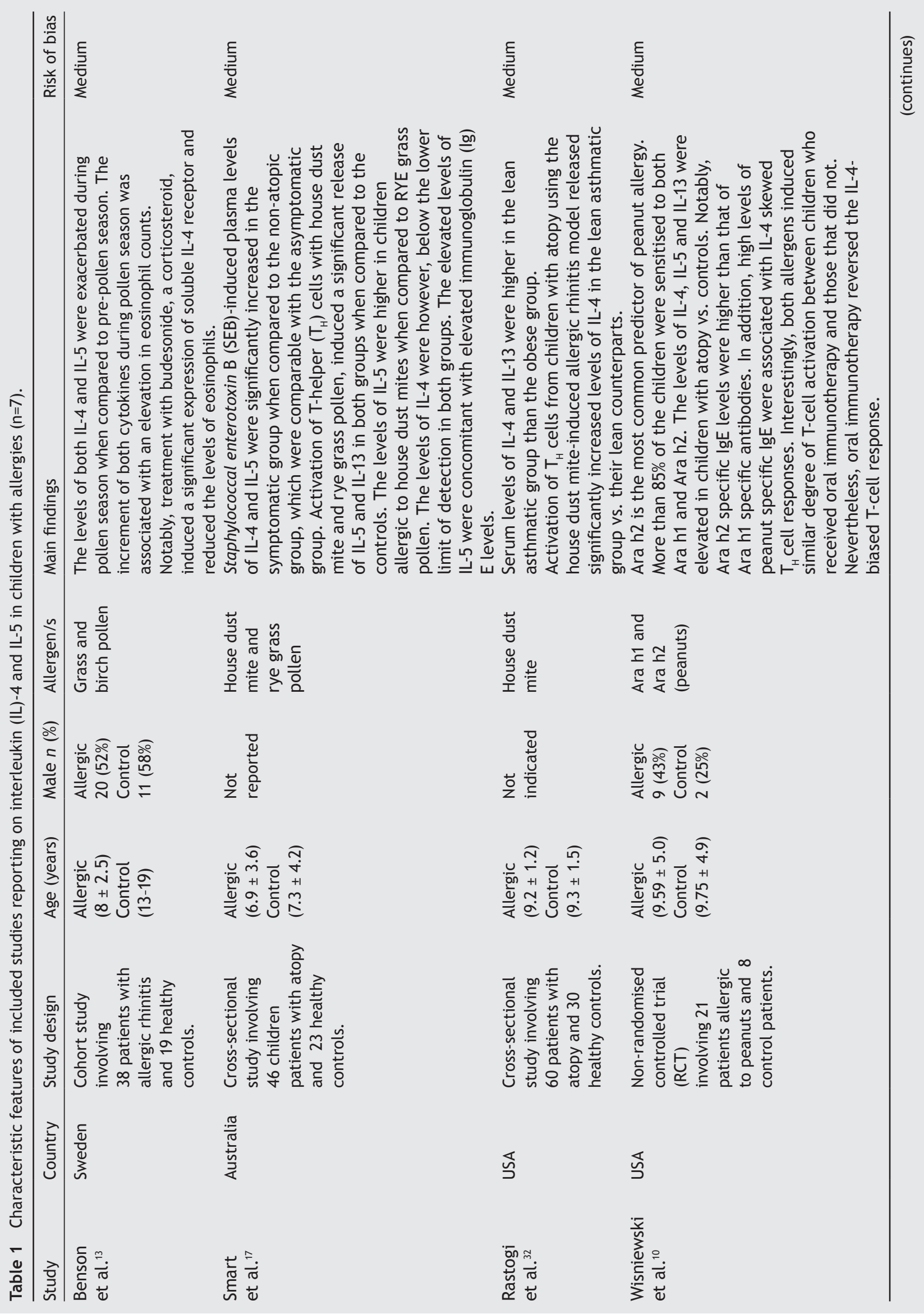




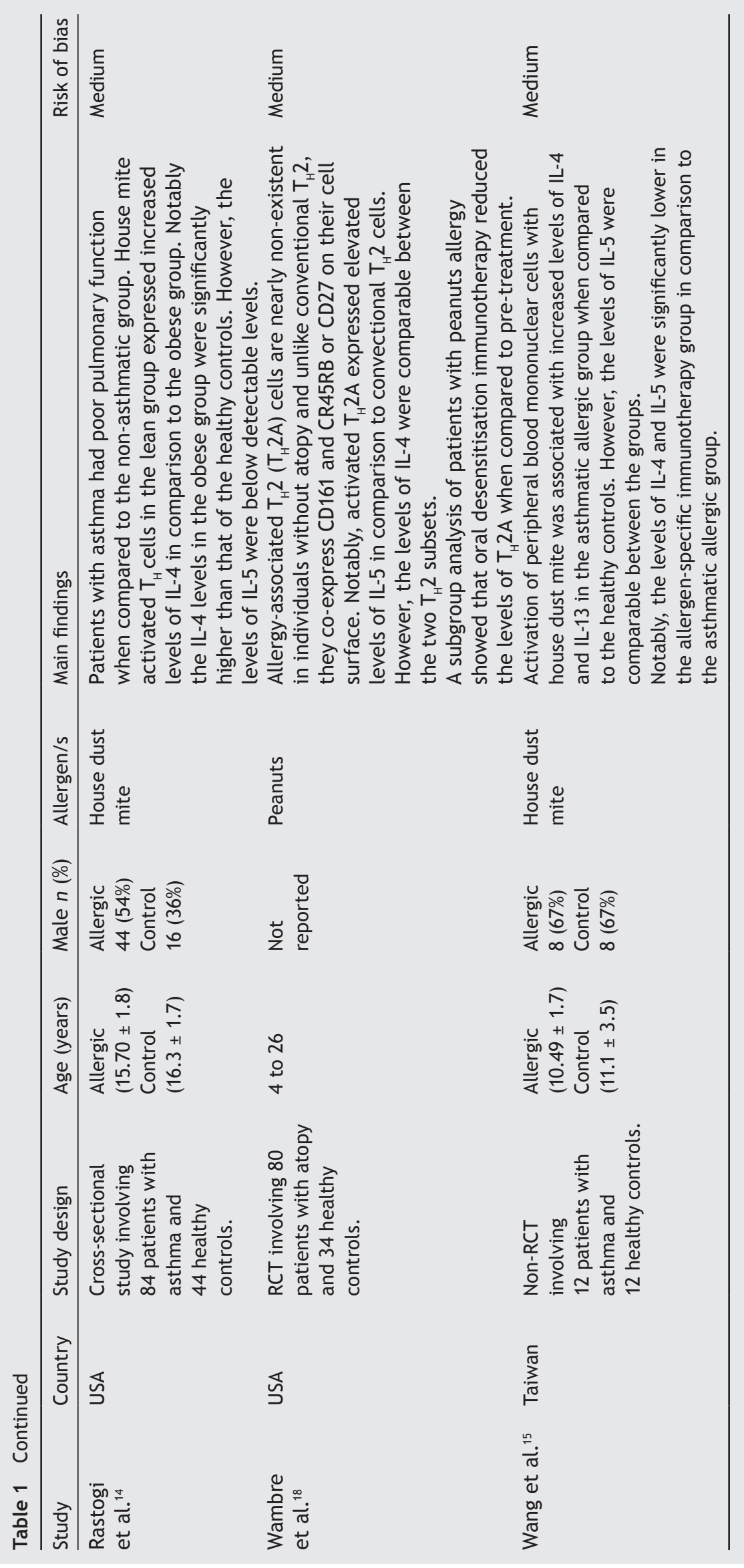




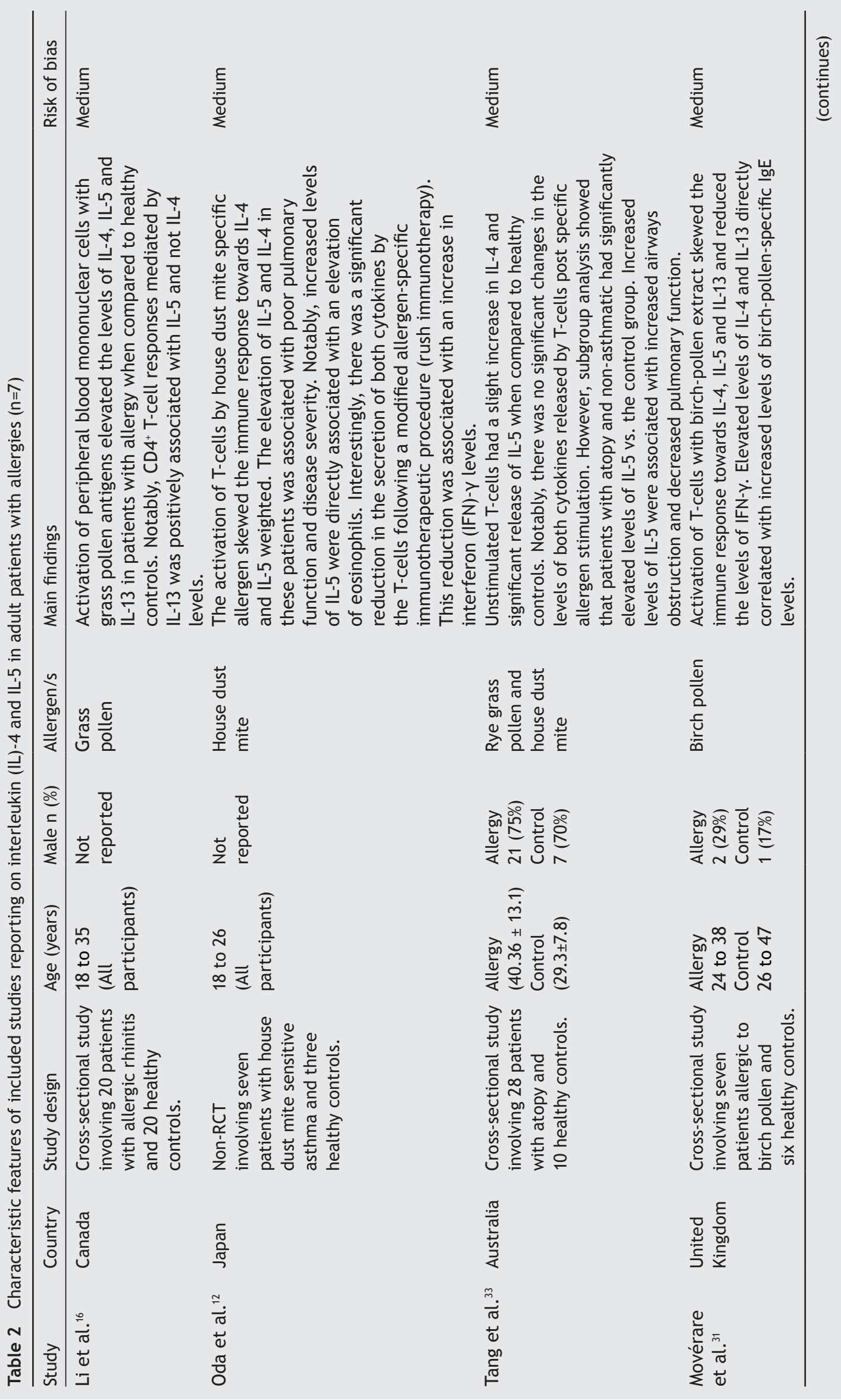




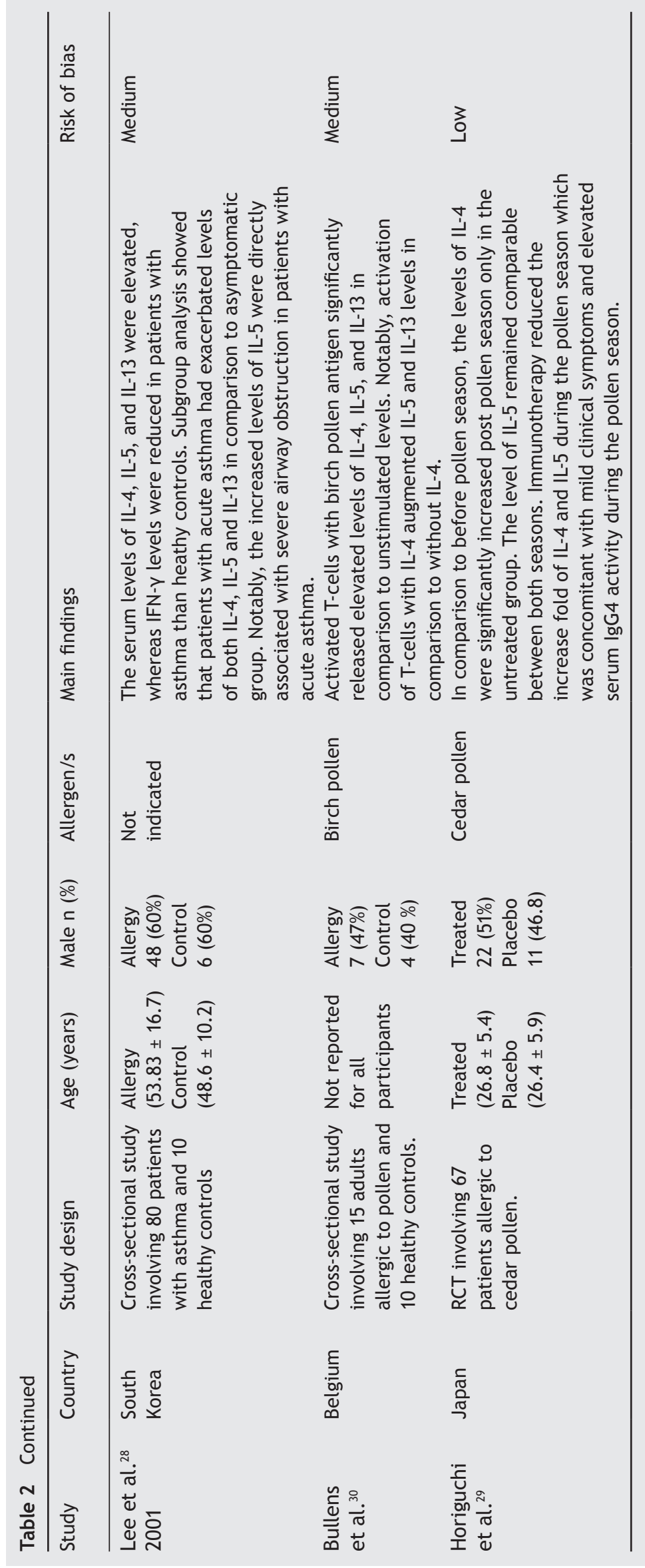




\section{The effect of treatment on the levels of IL-4 and IL-5 in patients with allergies}

As expected in allergic inflammation, a comparison of IL-4 levels between symptomatic patients and healthy controls showed a large effect size (Hedges' g: 1.30 [95\% $\mathrm{Cl}:-0.10,2.70], \mathrm{P}=0.068$ ) (Figure 2) whilst revealing a small effect size in IL-5 levels (Hedges' g: 0.38 [95\% $\mathrm{Cl}:-0.14,0.90], \mathrm{P}=0.150$ ) (Figure 3 ). Hence, treatment strategies are designed to antagonise the effect of these cytokines in allergic responses. A total of $43 \%$ of included studies reported on the effect of treatment on these $\mathrm{T}_{\mathrm{H}}$ 2-type cytokines. Treatment with corticosteroids, ${ }^{13}$ oral immunotherapy ${ }^{12,15,19}$ and rush immunotherapy reduced IL-4 and IL-5 levels. ${ }^{10,18}$ This decrease was associated with reduced eosinophil counts and increased levels of $T_{H}$ 1-type cytokines. Notably, treatment lowered IL-4 levels to almost like that of healthy controls (Hedges' g: 0.11 [95\% Cl: $-0.34,0.56], P=0.638$ ) (Figure 2). Only one study reported on the levels of IL-5 in the treated group, hence we could not perform a meta-analysis. Nonetheless, there was a large effect size in the levels of IL-5 between untreated and treated individuals with allergies (Hedges' g: 0.82 [95\% Cl: $-0.89,2.53$ ], $\mathrm{P}=0.346$ ) (Figure 3).

\section{Discussion}

This systematic review and meta-analysis aimed to comprehensively assess available literature reporting on $\mathrm{T}_{\mathrm{H}} 2$ mediated inflammation in allergic reactions. The primary objective of this study was to determine whether the levels of IL-4 and IL-5, the key cytokines that mediate inflammation in allergic reactions, are influenced by the type of allergen. Moreover, the secondary objective was to evaluate the effect of various treatment strategies on the levels of these cytokines. The pooled estimates showed that the levels of IL- 4 and IL- 5 are allergen-dependent. Notably, HDM elicited greater IL-4 and IL-5 mediated immune responses when compared to pollen. Moreover, treatment with the anti-inflammatory corticosteroid drugs or allergen-specific immunotherapy (rush immunotherapy) was effective in normalising the levels of $\mathrm{T}_{\mathrm{H}} 2$-cytokines and ameliorating the allergy-associated symptoms. ${ }^{10,13,15,18,30}$ the total nasal symptom scores (TNSS).

The secretion of IL- 4 and IL- 13 by activated $\mathrm{T}_{\mathrm{H}} 2$ cells drive the immune responses triggered by invading allergens in immediate reactions. The subsequent binding of IL- 4 and IL-13 to their respective receptors, IL-4Ra and IL-13Ra, activates Janus kinase/signal transducers and activator of transcription-6 (JAK/STAT6) signalling transduction pathway. ${ }^{35}$ This results in the activation of various promoter genes such as transforming growth factor-beta 1 (TGF-B1) and signalling pathways which are essential for the activation and differentiation of naïve T-cells into $\mathrm{T}_{H} 2$ and $\mathrm{B}$-cells to produce IgE antibodies. ${ }^{36-38}$ Notably, the IL-4 and IL-13-induced STAT6 signalling is enhanced in patients with atopic asthma compared to healthy individuals. ${ }^{39}$ The activation of STAT6 signalling in allergic responses is associated with increasing levels of IL-4 and IgE, and a decrease in $\mathrm{T}_{H} 1$ cytokines. ${ }^{39,40}$ Interestingly, in an animal study of asthma, STAT6 ${ }^{\text {null }}$ mice had reduced airway inflammation congruent with impaired $\mathrm{T}_{\mathrm{H}} 2$ differentiation and reduced $\mathrm{T}_{\mathrm{H}} 2$ immune responses. ${ }^{41,42}$ Although the studies included in our meta-analysis did not assess STAT signalling, our data synthesis showed that the levels of IL- 4 and IL-13 were increased in patients with allergy and are associated with increased allergen-specific

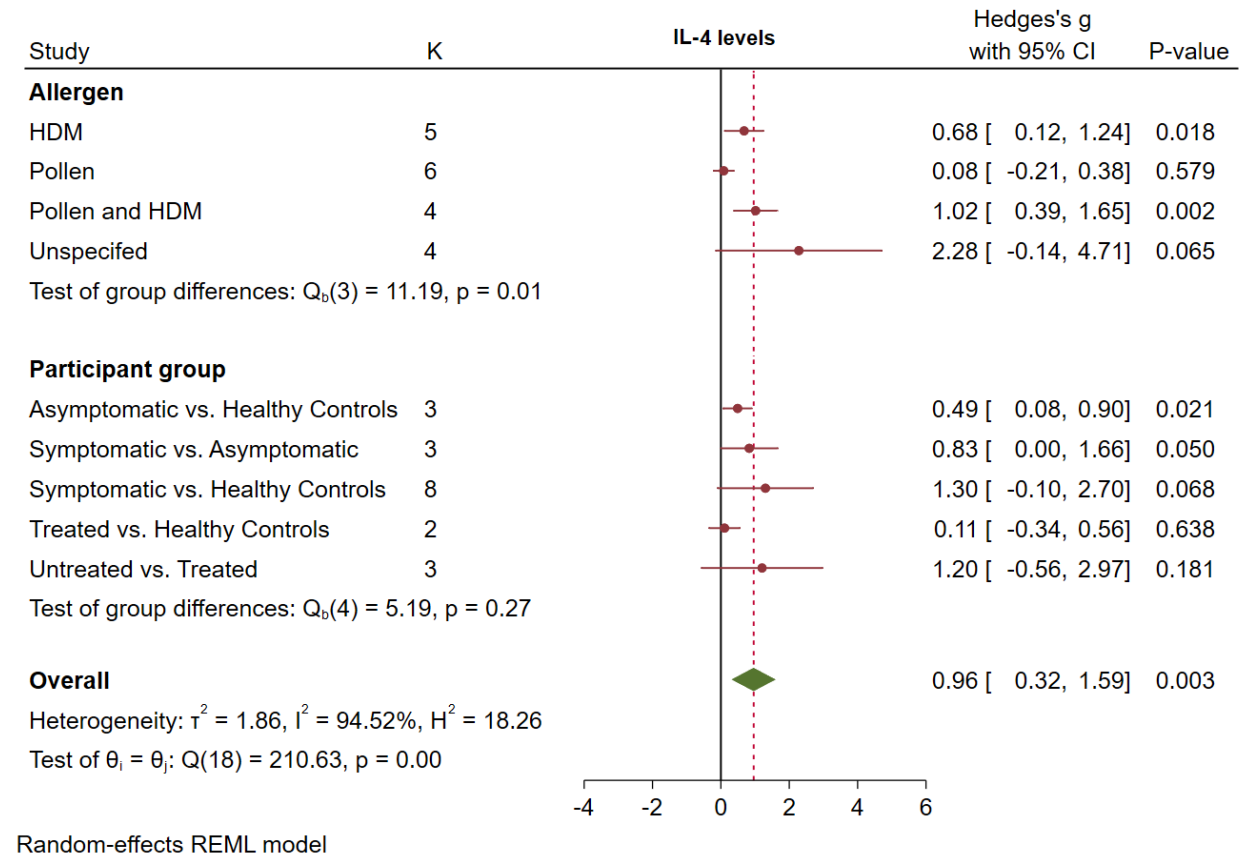

Figure 2 The levels of IL-4 in patients with allergies as an effect measure of $\mathrm{T}_{\mathrm{H}} 2$ immune response. 
IgE,and disease severity, ${ }^{10,12,31}$ and reduced IFN- $\gamma$ levels. ${ }^{28,31}$ This highlights the shift towards $T_{H} 2$ in the $T_{H} 1 / T_{H} 2$ paradigm in allergic responses, possibly induced by enhanced IL-4/IL13-mediated JAK/STAT6 signalling.

The increased activation of eosinophils is also associated and implicated in $\mathrm{T}_{\mathrm{H}} 2$ inflammation in allergic responses. ${ }^{43}$ Even though various cytokines such as IL-4 and IL-3 may induce the differentiation and activation of eosinophils, IL-5 also facilitates these processes. ${ }^{44}$ Notably, the binding of IL-5 to its receptor results in the downstream activation of the PI3K-AKT and MAPK signalling pathways, various STAT transcription factors and tyrosine kinases, which collectively promotes cell differentiation, activation and proliferation and inhibit apoptosis. ${ }^{45}$ As expected, we report on increased levels of IL-5 in allergic responses, which were concomitant with elevated eosinophil counts and increased airways obstruction. ${ }^{12,13,28,33}$

The use of allergen-specific immunotherapy and anti-inflammatory drugs in patients with allergies is effective in lowering the levels of allergy-associated $\mathrm{T}_{\mathrm{H}} 2$ cells, IL-4, IL-13 and IL-5. . $^{10,12,13,15,18,29}$ However, because of the heterogeneous nature of allergic responses and confounding factors such as obesity that alter immune responses, ${ }^{3}$ there is an increased interest in cytokine-targeting biologics as an alternative treatment strategy for allergic responses. ${ }^{11}$ In that context, while both IL-4 and IL-5 play pivotal roles in mediating type 2 inflammation in allergic patients, our meta-analysis showed that IL-4/IL-5 immune responses are weighted towards IL- 4 when compared to IL- 5 as indicated by a larger effect size in the levels of the former cytokine. Thus, cytokine-targeting biologics that directly antagonises IL-4/IL-13 may be effective in ameliorating allergic responses. Most importantly, for optimal therapeutic benefit, the type of antigen should be considered when determining dosage. However, it should be noted that utilisation of IL-4/IL-13 or IL-5 biologic antagonists should be done with caution as it requires a delicate balance in order to correct and maintain the $\mathrm{T}_{\mathrm{H}} 1 / \mathrm{T}_{\mathrm{H}} 2$ paradigm, as reported elsewhere. ${ }^{35,37}$

The main strength of our study is its uniqueness. To our knowledge, this is the first systematic review and meta-analysis to assess the effect of allergen type on the levels of IL-4 and IL-5 in patients with allergies. In addition, the comprehensive literature search and the subsequent data extraction were independently carried out by two reviewers, thus minimising the risk of errors. Moreover, the inter-rater reliability scores were high in the study selection and the risk of bias assessment. Even though the included studies had a medium risk of bias overall, the studies scored poor in the external validity domain. As a result, the findings must be adopted with caution in a population outside this study's populace. Nonetheless, the findings are a true representation of $\mathrm{T}_{\mathrm{H}} 2$ responses in allergies as denoted by a high score in internal validity. The main limitation of our study is that some of the included studies did not report on the exact levels of IL-4 and IL-5, so we estimated some levels from the reported graph values using the GetData Graph Digitizer software.

\section{Conclusion}

$\mathrm{T}_{\mathrm{H}}$ 2-mediated inflammation in patients with allergies is allergen dependent and treatment with anti-inflammatory corticosteroid drugs or allergen-specific immunotherapy is effective in normalising the levels of $\mathrm{T}_{\mathrm{H}} 2$-cytokines and ameliorating the allergy-associated symptoms. Therefore,

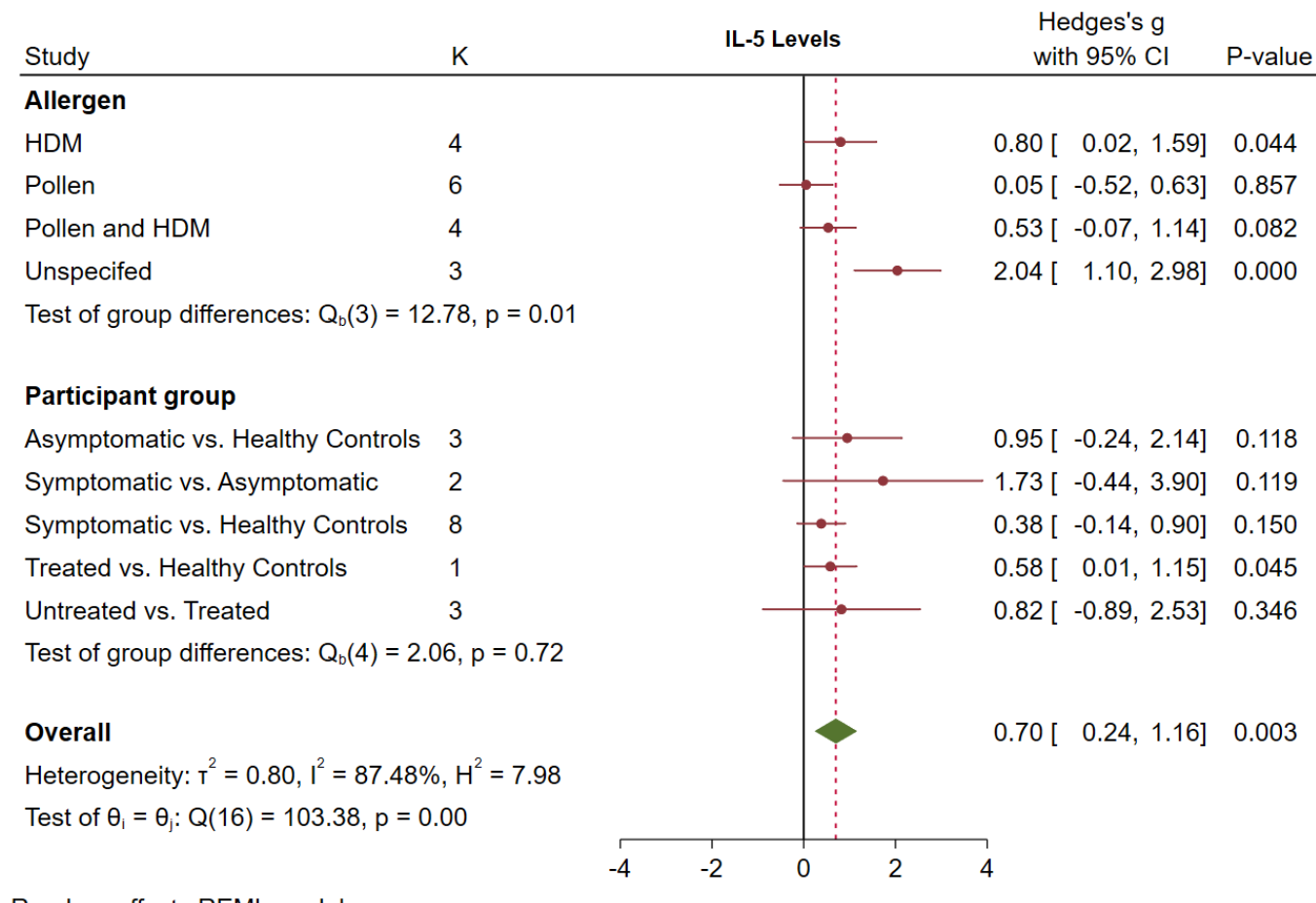

Figure 3 Effect estimates of $\mathrm{T}_{\mathrm{H}} 2$ immune response in patients with allergies measured by the levels of IL-5. 
to further improve the efficacy of treatment in allergic reactions, the type of allergen should be considered when planning therapeutic strategies, particularly those involving the usage of cytokine-targeting biologics that antagonise the activities of $\mathrm{T}_{\mathrm{H}} 2$ signature cytokines.

\section{Abbreviations}

IL: interleukin; cardiovascular diseases; JAK/STAT: Janus kinase/signal transducers and activator of transcription; TH2: T helper 2

\section{Declarations}

Ethics approval and consent to participate: Not applicable.

\section{Consent for publication: Not applicable}

Availability of data and materials: The authors confirm that the data supporting the findings of this study are available within the article and its supplementary files.

Conflict of interests: We declare no conflict of interests associated with this manuscript.

Funding: Not applicable

Authors' contribution: EPN, BBN and TMN conceptualised, designed and drafted this manuscript. EPN and TMN search strategy; EPN and FN - study selection; EPN, FN and TMN - data extraction; EPN, FN and PVD - study appraisal; EPN, BBN and TMN - statistical analysis, EPN, BBN, PVD, EN and TMN - editing and final approval of manuscript. TMN is the guarantor of this systematic review and meta-analysis Acknowledgments: BBN is partially funded by the National Research Foundation (NRF) of South Africa (Grant Number: 107519 to BB Nkambule). BBN is also a University of KwaZulu-Natal (UKZN) Developing Research Innovation, Localisation and Leadership in South Africa (DRILL) fellow. DRILL, is a NIH D43 grant (D43TW010131) awarded to UKZN in 2015 to support a research training and induction programme for early career academics. PV Dludla was partially supported as a Post-Doctoral Fellow by funding from Research Capacity Division of the South African Medical Research Council (SAMRC) through its division of Research Capacity Development under the Inta-Mural Post-Doctoral Fellowship Programme from funding received from the South African Treasury. The content hereof is the sole responsibility of the authors and do not necessary present the official views of SAMRC or the funders.

\section{References}

1. Kidd P. Th1/Th2 balance: the hypothesis, its limitations, and implications for health and disease. Altern Med Rev. 2003;8(3):223-46. Available from: https://pubmed.ncbi.nlm. nih.gov/12946237/

2. Singh VK, Mehrotra S, Agarwal SS. The paradigm of Th1 and Th2 cytokines: its relevance to aotoimmunity and allergy. Immunol Res. 1999;20(2):147-61. https://doi.org/10.1007/ BF02786470
3. Nyambuya TM, Dludla PV, Mxinwa V, Nkambule BB. Obesityrelated asthma in children is characterized by T-helper 1 rather than T-helper 2 immune response: a meta-analysis. Ann Allergy Asthma Immunol. 2020;125(4):425-32. https://doi. org/10.1016/j.anai.2020.06.020

4. Uzzaman A, Cho SH. Classification of hypersensitivity reactions. Allergy Asthma Proc. 2012;33(SUPPL 1):96-9. https:// doi.org/10.2500/aap.2012.33.3561

5. Justin Vaillant AA, Vashist R, Zito PM. Immediate hypersensitivity reactions - StatPearls - NCBI Bookshelf [Internet]. [Cited 2020 August16]. Treasure Island (FL): StatPearls Publishing; 2020.

6. Kubo M. T follicular helper and $\mathrm{TH} 2$ cells in allergic responses. Allergol Int. 2017;66(3):377-81. https://doi.org/10.1016/j. alit.2017.04.006

7. Chomarataand P, Banchereaua J. Interleukin- 4 and Interleukin-13: their Similarities and Discrepancies. Immunol Res. 1998;17(214):1-52. https://doi.org/10.3109/08830189809084486

8. Kouro T, Takatsu K. IL-5- and eosinophil-mediated inflammation: from discovery to therapy. Int Immunol. 2009;21(12):13039. https://doi.org/10.1093/intimm/dxp102

9. IL-4 and IL-5 regulate allergic response NFAT1 deficiency [Internet]. R \& D System . 2020. Available from: https://www.rndsystems.com/resources/articles / il-4-and-il-5-regulate-allergic-response-nfat1-deficiency

10. Wisniewski JA, Commins SP, Agrawal R, Hulse KE, Yu MD, Cronin $\mathrm{J}$, et al. Analysis of cytokine production by peanut-reactive $T$ cells identifies residual Th2 effectors in highly allergic children who received peanut oral immunotherapy. Clin Exp Allergy. 2015;45(7):1201-13. https://doi.org/10.1111/cea.12537

11. Lawrence MG, Steinke JW, Borish L. Cytokine-targeting biologics for allergic diseases. Ann Allergy Asthma Immunol. 2018;120(4):376-81. https://doi.org/10.1016/j.anai.2018.01.009

12. Oda N, Yamashita N, Minoguchi K, Takeno M, Kaneko S, Sakane $\mathrm{T}$, et al. Long-term analysis of allergen-specific $\mathrm{T}$ cell clones from patients with asthma treated with allergen rush immunotherapy. Cell Immunol. 1998;190(1):43-50. https://doi. org/10.1006/cimm.1998.1391

13. Benson M, Strannegård IL, Wennergren G, Strannegård Ö. Cytokines in nasal fluids from school children with seasonal allergic rhinitis. Pediatr Allergy Immunol. 1997;8(3):143-9. https://doi.org/10.1111/j.1399-3038.1997.tb00168.x

14. Rastogi D, Fraser S, Oh J, Huber AM, Schulman Y, Bhagtani $\mathrm{RH}$, et al. Inflammation, metabolic dysregulation, and pulmonary function among obese urban adolescents with asthma. Am J Respir Crit Care Med. 2015;191(2):149-60. https://doi. org/10.1164/rccm.201409-15870C

15. Wang CM, Chang CB, Chan MW, Wen ZH, and Wu SF. Dust mite allergen-specific immunotherapy increases IL4 DNA methylation and induces Der p-specific $\mathrm{T}$ cell tolerance in children with allergic asthma. Cell \& mole immunol. 2018;15(11):963972. https://doi.org/10.1038/cmi.2017.26

16. Li Y, Simons FE, Hayglass KT. Environmental antigen-induced IL-13 responses are elevated among subjects with allergic rhinitis, are independent of IL-4, and are inhibited by endogenous IFN- $\gamma$ Synthesis1. J Immunol. 1998 Nov;161(12):7007-14. Available from: https://pubmed.ncbi.nlm.nih.gov/9862737/

17. Smart JM, Tang MLK, Kemp AS. Polyclonal and allergen-induced cytokine responses in children with elevated immunoglobulin E but no atopic disease. Clin Exp Allergy. 2002;32(11):1552-7. https://doi.org/10.1046/j.1365-2222.2002.01532.x

18. Wambre E, Bajzik V, DeLong JH, O’Brien K, Nguyen QA, Speake $C$, et al. A phenotypically and functionally distinct human TH2 cell subpopulation is associated with allergic disorders. Sci Transl Med. 2017;9(401). https://doi.org/10.1126/ scitranslmed.aam9171

19. Larsen JN, Broge L, Jacobi H. Allergy immunotherapy: the future of allergy treatment. Drug Discov Today. 2016;21(1):2637. https://doi.org/10.1016/j.drudis.2015.07.010 
20. Mosen DM, Schatz M, Magid DJ, Camargo CA. The relationship between obesity and asthma severity and control in adults. J Allergy Clin Immunol. 2008;122(3). https://doi.org/10.1016/j. jaci.2008.06.024

21. Mennini M, Dahdah L, Fiocchi A. Two phase 3 trials of dupilumab versus placebo in atopic dermatitis. N Engl J Med. 2017;376(11):1090. https://doi.org/10.1056/NEJMc1700366

22. Castro M, Wenzel SE, Bleecker ER, Pizzichini E, Kuna P, Busse WW, et al. Benralizumab, an anti-interleukin 5 receptor a monoclonal antibody, versus placebo for uncontrolled eosinophilic asthma: a phase $2 \mathrm{~b}$ randomised dose-ranging study. Lancet Respir Med. 2014;2(11):879-90. https://doi.org/10.1016/ S2213-2600(14)70201-2

23. Shamseer L, Moher D, Clarke M, Ghersi D, Liberati A, Petticrew $M$, et al. Preferred reporting items for systematic review and meta-analysis protocols (prisma-p) 2015: Elaboration and explanation. BMJ. 2015;349:1-25. https://doi. org/10.1136/bmj.g7647

24. Ryan R, Synnot A, Prictor M, Hill S. Data extraction template for included studies. Cochrane Consumers Communication Group [Internet]. Version 1.8. [Updated 2016 Nov 29]. Available from: https://cccrg.cochrane.org , files , public , uploads

25. O'Connor SR, Tully MA, Ryan B, Bradley JM, Baxter GD, MCDonough SM. Failure of a numerical quality assessment scale to identify potential risk of bias in a systematic review: a comparison study. BMC Res Notes. 2015;8(1):1-7. https://doi. org/10.1186/s13104-015-1181-1

26. Landis JR, Koch GG. The measurement of observer agreement for categorical data. Biometrics. 1977;33(1):159-74. https:// doi.org/10.2307/2529310

27. Wan X, Wang W, Liu J, Tong T. Estimating the sample mean and standard deviation from the sample size, median, range and/or interquartile range. BMC Med Res Methodol. 2014;14(1):1-13. https://doi.org/10.1186/1471-2288-14-135

28. Lee YC, Lee KH, Lee HB, Rhee YK. Serum levels of interleukins (IL)-4, IL-5, IL-13, and interferon- $\gamma$ in acute asthma. J Asthma. 2001;38(8):665-71. https://doi.org/10.1081/JAS-100107544

29. Horiguchi S, Okamoto Y, Yonekura S, Okawa T, Yamamoto H, Kunii $\mathrm{N}$, et al. A randomized controlled trial of sublingual immunotherapy for japanese cedar pollinosis. Int Arch Allergy Immunol. 2008;146(1):76-84. https://doi.org/10.1159/000112506

30. Bullens DMA, Van Den Keybus C, Dilissen E, Kasran A, Ceuppens JL. Allergen-specific T cells from birch-pollen-allergic patients and healthy controls differ in T helper 2 cytokine and in interleukin-10 production. Clin Exp Allergy. 2004;34(6):87987. https://doi.org/10.1111/j.1365-2222.2004.01955.x

31. Movérare R, Elfman L, Stålenheim G, Björnsson E. Study of the Th1/Th2 balance, including IL-10 production, in cultures of peripheral blood mononuclear cells from birch-pollen-allergic patients. Allergy Eur J Allergy Clin Immunol. 2000;55(2):171-5. https://doi.org/10.1034/j.1398-9995.2000.00244.x

32. Rastogi D, Canfi SM, Andrade A, Isasi CR, Hall C, Rubinstein A, et al. Obesity-associated asthma in children: a distinct entity. Chest. 2012;141(4):895-905. https://doi.org/10.1378/ chest.11-0930
33. Tang C, Rolland JM, Ward C, Li X, Gollant S, Walters EH. Differential regulation of allergen- specific TH2 - but not TH1type responses by alveolar macrophages in atopic asthma. J Allerg Clin Immunol. 1998;102(3):368-75. https://doi. org/10.1016/S0091-6749(98)70122-8

34. Bao K, Reinhardt RL. The differential expression of IL-4 and IL-13 and its impact on type-2 immunity. Cytokine. 2015;75(1):25-37. https://doi.org/10.1016/j.cyto.2015.05.008

35. Kips JC, Tournoy KG, Pauwels RA. New anti-asthma therapies: suppression of the effect of interleukin (IL)- 4 and IL-5. Eur Respir J. 2001;17(3):499-506. https://doi.org/10.1183/0903193 6.01.17304990

36. Shimoda H, Van Deursen J, Sangster MY, Sarawar SR, Carson RT, Tripp RA, et al. Lack of IL-4-induced Th2 response and IgE class switching in mice with disrupted Stat6 gene. Nature. 1996;380(6575):630-3. https://doi.org/10.1038/380630a0

37. Junttila IS. Tuning the cytokine responses: an update on interleukin (IL)-4 and IL-13 receptor complexes. Front Immunol. 2018;9:888. https://doi.org/10.3389/fimmu.2018.00888

38. Kuperman DA, Schleimer RP. Interleukin-4, Interleukin-13, signal transducer and activator of transcription factor 6 , and allergic asthma. Curr Mol Med. 2008;8(5):384-92. https://doi. org/10.2174/156652408785161032

39. Antczak A, Domańska-Senderowska D, Górski P, PastuszakLewandoska D, Nielepkowicz-Goździńska A, Szewczyk K, et al. Analysis of changes in expression of IL-4/IL-13/ STAT6 pathway and correlation with the selected clinical parameters in patients with atopic asthma. Int J Immunopathol Pharmacol. 2016;29(2):195-204. https://doi. org/10.1177/0394632015623794

40. Gernez Y, Tirouvanziam R, Nguyen KD, Herzenberg LA, Krensky AM, Nadeau KC. Altered phosphorylated signal transducer and activator of transcription profile of CD4+CD161+ T cells in asthma: Modulation by allergic status and oral corticosteroids. J Allergy Clin Immunol. 2007;120(6):1441-8. https:// doi.org/10.1016/j.jaci.2007.08.012

41. Chapoval SP, Dasgupta P, Smith EP, DeTolla LJ, Lipsky MM, Kelly-Welch AE, et al. STAT6 expression in multiple cell types mediates the cooperative development of allergic airway disease. J Immunol. 2011;186(4):2571-83. https://doi. org/10.4049/jimmunol.1002567

42. Kuperman BD, Schofield B, Wills-karp M, Grusby MJ. Airway Hyperresponsiveness and mucus production. J Exp Med. 1998;187(6):939-48. https://doi.org/10.1084/jem.187.6.939

43. Kita H. Eosinophils: multificated biologic properties and roles in health and disease. Immunol Rev. 2011;242(1):161-77. https://doi.org/10.1111/j.1600-065X.2011.01026.x

44. Greenfeder S, Umland SP, Cuss FM, Chapman RW, Egan RW. Th2 cytokines and asthma. The role of interleukin- 5 in allergic eosinophilic disease. Respir Res. 2001;2(2):71-9. https://doi. org/10.1186/rr41

45. Kandasamy K, Mohan SS, Raju R, Keerthikumar S, Kumar GSS, Venugopal AK, et al. IL-5 Signaling Pathway (Homo sapiens) [Internet]. [Updated 2021 May 9]. Availble from https://www. wikipathways.org/index.php/Pathway:WP127. 


\section{Supplementary}

Table S1 Search strategies

\begin{tabular}{ll}
\hline Terms & Search terms \\
\hline \#1 & (T helper cells [MeSH Terms]) =45,274 hits \\
\#2 & (IL-5[MeSH Terms])=5,938 hits \\
\#3 & $($ IL-4[MeSH Terms]) 22,802 hits \\
\#4 & (allergen [MeSH Terms] $=41,348$ hits \\
Combined \#1 and & $(((T$ helper cells[MeSH Terms]) AND (IL-5[MeSH Terms])) OR (IL-4[MeSH Terms])) AND (allergen[MeSH \\
\#2 or \#3 and \#4 & Terms]) \\
& $=\mathbf{4 6}$ hits (Age: $2-12$ years) \\
Combined \#1 and & $(((T$ helper cells[MeSH Terms]) AND (IL-5[MeSH Terms])) AND (IL-4[MeSH Terms])) AND (allergen[MeSH \\
\#2 and \#3 and \#4 & Terms]) \\
& $=67$ hits
\end{tabular}

Table S2 Modified Downs and Black check list scores for included studies $(n=14)$

\begin{tabular}{|c|c|c|c|c|c|c|c|c|c|c|c|c|c|c|c|}
\hline Author & Domain & $\begin{array}{c}\text { Benson } \\
1997\end{array}$ & $\begin{array}{c}\text { Smart } \\
2002\end{array}$ & $\begin{array}{c}\text { Rastogi } \\
2012\end{array}$ & $\begin{array}{c}\text { Wisneiwski } \\
2015\end{array}$ & $\begin{array}{l}\text { Rastogi } \\
2015\end{array}$ & $\begin{array}{c}\text { Wambre } \\
2017\end{array}$ & $\begin{array}{c}\text { Wang } \\
2018\end{array}$ & $\begin{array}{c}\mathrm{Li} \\
1998\end{array}$ & $\begin{array}{c}\text { Oda } \\
1998\end{array}$ & $\begin{array}{l}\text { Tang } \\
1998\end{array}$ & $\begin{array}{c}\text { Moverare } \\
2000\end{array}$ & $\begin{array}{l}\text { Lee } \\
2001\end{array}$ & $\begin{array}{c}\text { Bullens } \\
2004\end{array}$ & $\begin{array}{l}\text { Horiguchi } \\
2008\end{array}$ \\
\hline \multirow{9}{*}{$\begin{array}{l}\text { Reporting } \\
\text { bias }\end{array}$} & 1 & 1 & 1 & 1 & 1 & 1 & 1 & 1 & 1 & 1 & 1 & 1 & 1 & 1 & 1 \\
\hline & 2 & 1 & 1 & 1 & 1 & 1 & 1 & 1 & 1 & 1 & 1 & 1 & 1 & 1 & 1 \\
\hline & 3 & 1 & 1 & 1 & 1 & 0 & 0 & 0 & 1 & 1 & 1 & 1 & 1 & 0 & 1 \\
\hline & 4 & 1 & 1 & 1 & 1 & 1 & 1 & 1 & 1 & 1 & 1 & 1 & 1 & 1 & 1 \\
\hline & 5 & 1 & 1 & 1 & 1 & 1 & 1 & 1 & 1 & 1 & 1 & 1 & 1 & 1 & 1 \\
\hline & 8 & 0 & 0 & 0 & 1 & 1 & 0 & 0 & 0 & 0 & 0 & 0 & 0 & 0 & 1 \\
\hline & 9 & 0 & 0 & 0 & 0 & 0 & 0 & 1 & 0 & 0 & 0 & 0 & 0 & 0 & 1 \\
\hline & 10 & 0 & 1 & 1 & 0 & 1 & 1 & 1 & 0 & 0 & 0 & 0 & 1 & 1 & 1 \\
\hline & Score & 7 & 8 & 8 & 8 & 8 & 7 & 8 & 7 & 6 & 7 & 7 & 8 & 7 & 10 \\
\hline \multirow{2}{*}{$\begin{array}{l}\text { External } \\
\text { validity }\end{array}$} & 11 & 1 & 1 & 1 & 1 & 0 & 0 & 1 & 0 & 1 & 1 & 1 & 0 & 1 & 1 \\
\hline & 12 & 0 & 0 & 0 & 0 & 0 & 0 & 0 & 0 & 0 & 0 & 1 & 1 & 0 & 1 \\
\hline \multirow{7}{*}{$\begin{array}{l}\text { Internal } \\
\text { validity }\end{array}$} & 15 & 0 & 0 & 0 & 0 & 0 & 1 & 0 & 0 & 0 & 0 & 0 & 0 & 0 & 1 \\
\hline & 16 & 1 & 1 & 1 & 1 & 1 & 1 & 1 & 1 & 1 & 1 & 1 & 1 & 1 & 1 \\
\hline & 17 & 0 & 0 & 0 & 0 & 0 & 1 & 1 & 1 & 1 & 1 & 1 & 0 & 1 & 1 \\
\hline & 18 & 1 & 1 & 1 & 1 & 1 & 1 & 1 & 1 & 1 & 1 & 1 & 1 & 1 & 1 \\
\hline & 19 & 0 & 1 & 1 & 1 & 0 & 0 & 0 & 0 & 0 & 0 & 0 & 1 & 0 & 0 \\
\hline & 20 & 1 & 1 & 1 & 1 & 1 & 1 & 1 & 1 & 1 & 1 & 1 & 1 & 1 & 1 \\
\hline & Score & 3 & 4 & 5 & 4 & 3 & 6 & 4 & 4 & 4 & 4 & 4 & 4 & 4 & 6 \\
\hline Selection & 21 & 1 & 1 & 1 & 1 & 1 & 1 & 1 & 1 & 1 & 1 & 1 & 1 & 1 & 1 \\
\hline \multirow[t]{4}{*}{ bias } & 22 & 1 & 1 & 1 & 1 & 1 & 0 & 0 & 1 & 1 & 0 & 0 & 0 & 1 & 1 \\
\hline & 23 & 0 & 0 & 0 & 0 & 0 & 1 & 0 & 0 & 0 & 0 & 0 & 0 & 0 & 1 \\
\hline & 24 & 0 & 0 & 0 & 0 & 0 & 1 & 0 & 0 & 0 & 0 & 0 & 0 & 0 & 1 \\
\hline & 25 & 0 & 1 & 1 & 0 & 1 & 1 & 1 & 1 & 1 & 1 & 1 & 1 & 0 & 1 \\
\hline
\end{tabular}


Table S3 Comparisons of standardised means of interleukin 4 and 5 levels based on the type of allergen

\begin{tabular}{lcrr}
\hline Allergens & Standardised means differences & $95 \% \mathrm{Cl}$ & $\mathrm{p}$-value \\
\hline Interleukin 4 Levels & & 0.02 to 1.18 & 0.0430 \\
HDM vs Pollen & 0.60 & -0.02 to 1.90 & 0.0009 \\
Pollen and HDM vs Pollen & 0.94 & 1.07 to 3.33 & 0.0002 \\
Unspecified vs Pollen & 2.20 & 0.57 to 2.63 & 0.0024 \\
HDM vs Unspecified & 1.60 & -0.02 to 1.90 & 0.0544 \\
Pollen and HDM vs Unspecified & 0.94 & -0.50 to 1.18 & 0.4232 \\
HDM vs Pollen and HDM & 0.34 & & 0.0878 \\
Interleukin 5 levels & & -0.11 to 1.61 \\
HDM vs Pollen & 0.75 & -0.21 to 1.17 & 0.1730 \\
Pollen and HDM vs Pollen & 0.48 & 1.40 to 2.57 & $<0.0001$ \\
Unspecified vs Pollen & 1.99 & 0.32 to 2.16 & 0.0086 \\
HDM vs Unspecified & 1.24 & 0.75 to 2.28 & 0.0001 \\
Pollen and HDM vs Unspecified & 1.51 & -1.26 to 0.72 & 0.5923 \\
HDM vs Pollen and HDM & -0.27 & & \\
\hline
\end{tabular}

Table S4 Sensitivity analysis of interleukin 4 and 5 levels based on study design

\begin{tabular}{lcccc}
\hline Study design & Number of studies & Omitted studies & Hedges's g [95\% Cl] & p-value \\
\hline Interleukin 4 & & 0 & $0.96[0.32,1.59]$ & 0.003 \\
All & 8 & 2 & $1.54[-0.31,3.40]$ & 0.103 \\
Cross-sectional & 6 & 7 & $0.18[-0.34,0.90]$ & 0.619 \\
Cohort & 1 & 7 & $1.00[0.19,1.80]$ & 0.016 \\
Non-RCT & 1 & & & 0.003 \\
Interleukin 5 & & 0 & $0.24,1.16]$ & 0.034 \\
All & 8 & 2 & $-0.96[-1.70,-0.24]$ & 0.012 \\
Cross-sectional & 6 & 7 & $0.57[-0.21,1.34]$ \\
Cohort & 1 & 7 & 0.151 \\
Non-RCT & 1 & &
\end{tabular}

